Research Article

\title{
Stability of solutions to some abstract evolution equations with delay
}

\author{
Nguyen S. Hoang ${ }^{1}$, Alexander G. Ramm ${ }^{2, *}$ \\ ${ }^{1}$ Department of Mathematics, University of West Georgia, Carrollton, GA 30116, USA \\ ${ }^{2}$ Department of Mathematics, Kansas State University, Manhattan, KS 66506, USA
}

(Received: 13 January 2021. Received in revised form: 26 January 2021. Accepted: 26 January 2021. Published online: 30 January 2021.)

(C) 2021 the authors. This is an open access article under the CC BY (International 4.0) license (www.creativecommons.org/licenses/by/4.0/).

\begin{abstract}
The global existence and stability of the solution to the delay differential equation (*) $\dot{u}=A(t) u+G(t, u(t-\tau))+f(t), t \geq 0$, $u(t)=v(t),-\tau \leq t \leq 0$, are studied. Here $A(t): \mathcal{H} \rightarrow \mathcal{H}$ is a closed, densely defined, linear operator in a Hilbert space $\mathcal{H}$ and $G(t, u)$ is a nonlinear operator in $\mathcal{H}$ continuous with respect to $u$ and $t$. We assume that the spectrum of $A(t)$ lies in the half-plane $\operatorname{Re} \lambda \leq \gamma(t)$, where $\gamma(t)$ is not necessarily negative and $\|G(t, u)\| \leq \alpha(t)\|u\|^{p}, p>1, t \geq 0$. Sufficient conditions for the solution to the equation to exist globally, to be bounded and to converge to zero as $t$ tends to $\infty$, under the non-classical assumption that $\gamma(t)$ can take positive values, are proposed and justified.
\end{abstract}

Keywords: abstract evolution problems; delay; stability; differential inequality; global existence.

2020 Mathematics Subject Classification: 34G20, 37L05, 44J05, 47J35.

\section{Introduction}

Consider the following delay differential equation

$$
\begin{aligned}
\dot{u} & =A(t) u+G(t, u(t-\tau))+f(t), \quad t \geq 0, \quad \dot{u}:=\frac{d u}{d t}, \\
u(t) & =v(t), \quad-\tau \leq t \leq 0, \quad \tau=\text { const }>0, \quad v(t) \in C([-\tau, 0] ; \mathcal{H}) .
\end{aligned}
$$

Reference to Equation (1) means reference to both Equations (1a) and (1b). Here, $A(t): \mathcal{H} \rightarrow \mathcal{H}$ is a closed, densely defined, linear operator in a Hilbert space $\mathcal{H}$ for any fixed $t \geq 0$,

$$
\operatorname{Re}\langle u, A(t) u\rangle \leq \gamma(t)\|u\|^{2}, \quad u \in \operatorname{Dom}(A) \subset \mathcal{H},
$$

$G(t, u)$ is a nonlinear operator in $\mathcal{H}$ for any fixed $t \geq 0$,

$$
\|G(t, u)\| \leq \alpha(t)\|u\|^{p}, \quad p>1, \quad u \in \mathcal{H},
$$

and $f(t)$ is a function on $\mathbb{R}_{+}=[0, \infty)$ with values in $\mathcal{H}$,

$$
\|f(t)\| \leq \beta(t), \quad t \geq 0 .
$$

Here, $\langle\cdot, \cdot\rangle$ and $\|\cdot\|$ denote the inner product and the norm in $\mathcal{H}$, respectively. The functions $\gamma(t)$ and $\alpha(t)$ are continuous on $[0, \infty)$ and real-valued.

Functional differential equations have been studied extensively in the literature (see, e.g., [1]- [5] and references therein). The usual assumption to derive the global existence and the stability of the solution to Equation $(1)$ is: $\gamma(t) \leq$ $\gamma_{0}<0, \forall t \geq 0$. If $A(t)$ is a square matrix, then the condition $\gamma(t) \leq \gamma_{0}<0$ implies that all the eigenvalues of $A(t)$ lie in the half-plane $\operatorname{Re} \lambda \leq \gamma_{0}<0$. In [6], [7], and [8], stability of solution to abstract differential Equation (1) when $\tau=0$, i.e., without delay, was studied for the cases $0<\gamma(t) \searrow 0$ and $0>\gamma(t) \nearrow 0$. The main tool for the study of the stability in [6], [7], and [8] under these non-classical assumptions is some nonlinear inequalities. These inequalities were also used in the study of the Dynamical Systems Method (DSM) for solving operator equations in [10]. In [4] the stability of the solution to Equation (1) with $\tau=0$, i.e., without delay, was studied for the case when $\gamma(t)$ can take positive and negative values. In [4] the nonlinear inequalities were not used, in contrast to [6], [7], and [8]. Using a nonlinear inequality with delay, the global existence and stability of Equation (1) were studied in [9] for the case when $f(t)=0$ and $G(t, u)$ is of the form $B(t) F(t, u)$ under the non-classical assumption that (2) holds but the inequality $\gamma(t) \leq \gamma_{0}<0$ does not hold for 
any $\gamma_{0}<0$. In this paper, we are interested in having the stability results for the solution to Equation (1) without using nonlinear inequalities similar to those used in [9].

A common approach to obtain the global existence of the solution to Equation (1) is to estimate $\|u(t)\|$ for $t \geq 0$ and use the local existence of the solution to extend the existence of the solution to $[0, \infty)$. An estimate of $\|u(t)\|$ for $t \geq 0$ can be derived from a nonlinear inequality (see inequality (7) below) which is obtained from Equation (1) as follows. Take the inner product of both sides of Equation (1a) with $u$ to get

$$
\begin{aligned}
\langle u(t), \dot{u}(t)\rangle & =\langle u(t), A(t) u(t)+G(t, u(t-\tau))+f(t)\rangle \\
& =\langle u(t), A(t) u(t)\rangle+\langle u(t), G(t, u(t-\tau))\rangle+\langle u(t), f(t)\rangle, \quad t \geq 0 .
\end{aligned}
$$

Denote $g(t):=\|u(t)\|$, take the real part of Equation (5) and use the triangle inequality, the Cauchy-Schwarz inequality, and inequalities (2)-(4) to get

$$
\dot{g}(t) g(t) \leq \gamma(t) g^{2}(t)+\alpha(t) g(t) g^{p}(t-\tau)+g(t) \beta(t), \quad t \geq 0 .
$$

The derivative $\dot{g}(t)$ in (6) is understood as the right derivative at $t$ if $g(t)=0$. From inequality (6) and Equation (1b) one gets

$$
\begin{aligned}
& \dot{g}(t) \leq \gamma(t) g(t)+\alpha(t) g^{p}(t-\tau)+\beta(t), \quad t \geq 0, \\
& g(t)=w(t):=\|v(t)\|, \quad-\tau \leq t \leq 0 .
\end{aligned}
$$

If $g(t)>0, \forall t \geq 0$, then it is clear that (7a) follows from (6). If $g(t)=0$ for some $t>0$, then these $t$ form a set of isolated points by the uniqueness theorem and therefore equations (6) and (7a) are equivalent. This equivalence is proved differently in Lemma 2.1 below. By studying the global existence and boundedness of a solution $g(t)$ to inequality (7) and using the local existence of the solution $u(t)$ to Equation (1), one can obtain the global existence and boundedness of $u(t)$.

In [9] the stability of the solution to the following differential equation with delay was studied:

$$
\begin{aligned}
\dot{u} & =A(t) u+B(t) F(t, u(t-\tau)), \quad t \geq 0, \quad \dot{u}:=\frac{d u}{d t}, \\
u(t) & =v(t), \quad-\tau \leq t \leq 0, \quad \tau=\text { const }>0 .
\end{aligned}
$$

It was assumed in [9] that $A(t)$ and $B(t)$ are linear operators in a Hilbert space $\mathcal{H}$ and $F(t, u)$ is a nonlinear operator in $\mathcal{H}$ for any fixed $t \geq 0$. It was also assumed in [9] that relation (2) holds, $\|B(t)\| \leq b(t)$, and $\|F(t, u)\| \leq \alpha(t,\|u\|)$. It is clear that Equation (8) is a special case of Equation (1) when the function $f(t)$ vanishes and $G(t, u)=B(t) F(t, u)$. Using the assumptions on $A(t), B(t)$, and $F(t, u)$ and an assumption on the existence of a local solution to problem (8), the following result was proved in [9]:

If there exists a function $\mu(t)>0$, defined for all $t \geq-\tau$, such that

$$
\begin{aligned}
b(t) \alpha\left(t, \frac{1}{\mu(t-\tau)}\right) \mu(t) & \leq-\gamma(t)-\frac{\dot{\mu}(t)}{\mu(t)}, \quad t \geq 0, \\
\|u(t)\| & \leq \frac{1}{\mu(t)}, \quad t \in[-\tau, 0],
\end{aligned}
$$

then the solution to (8) exists for all $t \geq 0$ and

$$
\|u(t)\| \leq \frac{1}{\mu(t)}, \quad t \geq 0 .
$$

Using this result the global existence and the boundedness of the solution to Equation (8) were obtained for some classes of functions $b(t), \gamma(t)$, and $\alpha(t, y)$ (see [9]).

Although the mentioned result in [9] is quite general, it requires to find a function $\mu(t)$ which solves inequality (9). In general, it is not easy to find such function $\mu(t)$ if the functions $b(t), \gamma(t)$, and $\alpha(t, y)$ are not simple.

In this paper we are interested in having stability results for Equation (1) under non-classical assumptions on the operator $A(t)$, namely that the function $\gamma(t)$ in (2) may change signs. In particular, we want find sufficient conditions on the functions $\gamma(t), \alpha(t)$, and $\beta(t)$ (see (2)-(4)) which yield the global existence and the boundedness of the solution to Equation (1).

The main results of this paper are Theorem 2.1, Theorem 2.2, and Corollary 2.1. In Theorems 2.1 and 2.2 sufficient conditions on $\gamma(t), \alpha(t)$, and $\beta(t)$ for the solution of Equation (1) to exist globally, to be bounded, and to decay to zero as $t \rightarrow \infty$, are given. A direct consequence of Theorem 2.1 for the case $f=0$ is formulated in Corollary 2.1. The novelties of our results compared to those in [9] include: 
Our results do not require one to find the function $\mu(t)>0$ which solves the nonlinear inequality (9).

Thus, these results are applicable when the functions $\gamma(t), \alpha(t)$, and $\beta(t)$ are quite general. Moreover, our results cover the case when $f \neq 0$ which was not considered in [9].

Throughout this paper, we suppose that the following assumption holds:

Assumption A: The equation

$$
\dot{u}=A(t) u+G(t, u(t-\tau))+f(t), \quad t \geq a, \quad \dot{u}:=\frac{d u}{d t}, \quad u(t)=v_{a}(t), \quad a-\tau \leq t \leq a, \quad v_{a} \in C([a-\tau, a] ; \mathcal{H}),
$$

has a unique local solution for all $a \geq 0$ and $v_{a} \in C([a-\tau, a] ; \mathcal{H})$.

It is known that Assumption A holds if $A(t)$ is a generator of a $C_{0}$ semigroup and the functions $\alpha(t)$ and $\beta(t)$ are continuous and bounded on $[0, \infty)$ (see, e.g., [1]).

\section{Main results}

Let us first show that inequalities (6) and (7) are equivalent.

Lemma 2.1. Let $g(t) \geq 0$ be a solution to

$$
\begin{aligned}
g(t) \dot{g}(t) & \leq \gamma(t) g^{2}(t)+\alpha(t) g(t) g^{p}(t-\tau)+g(t) \beta(t), \quad t \geq 0, \\
g(t) & =w(t) \geq 0, \quad-\tau \leq t \leq 0 .
\end{aligned}
$$

Then $g(t)$ solves the inequality

$$
\begin{aligned}
& \dot{g}(t) \leq \gamma(t) g(t)+\alpha(t) g^{p}(t-\tau)+\beta(t), \quad t \geq 0, \\
& g(t)=w(t), \quad-\tau \leq t \leq 0 .
\end{aligned}
$$

Proof. Equation (10a) can be written as

$$
\frac{d}{d t} g^{2}(t) \leq 2 \gamma(t) g^{2}(t)+2 \alpha(t) g(t) g^{p}(t-\tau)+2 g(t) \beta(t), \quad t \geq 0 .
$$

Let

$$
g_{\epsilon}(t):=\left[g^{2}(t)+\epsilon e^{2 \int_{0}^{t} \gamma(\xi) d \xi}\right]^{\frac{1}{2}}, \quad t \geq-\tau, \quad \epsilon=\text { const }>0 .
$$

From (12) and (13) one gets

$$
\begin{aligned}
\frac{d}{d t} g_{\epsilon}^{2}(t) & =\frac{d}{d t} g^{2}(t)+2 \epsilon \gamma(t) e^{2 \int_{0}^{t} \gamma(\xi) d \xi} \\
& \leq 2 \gamma(t) g^{2}(t)+2 \alpha(t) g(t) g^{p}(t-\tau)+2 g(t) \beta(t)+2 \epsilon \gamma(t) e^{2 \int_{0}^{t} \gamma(\xi) d \xi} \\
& =2 \gamma(t) g_{\epsilon}^{2}(t)+2 \alpha(t) g(t) g^{p}(t-\tau)+2 g(t) \beta(t) \\
& \leq 2 \gamma(t) g_{\epsilon}^{2}(t)+2 \alpha(t) g_{\epsilon}(t) g_{\epsilon}^{p}(t-\tau)+2 g_{\epsilon}(t) \beta(t), \quad t \geq 0 .
\end{aligned}
$$

Here the inequality $g_{\epsilon}(t)>g(t), \forall t \geq-\tau, \epsilon>0$, was used. Inequality (14) implies

$$
g_{\epsilon}(t) \dot{g}_{\epsilon}(t) \leq \gamma(t) g_{\epsilon}^{2}(t)+\alpha(t) g_{\epsilon}(t) g_{\epsilon}^{p}(t-\tau)+g_{\epsilon}(t) \beta(t), \quad t \geq 0 .
$$

Since $g_{\epsilon}(t) \geq \sqrt{\epsilon} \int_{0}^{t} \gamma(\xi) d \xi>0, \forall t \geq 0, \epsilon>0$, by (13), it follows from inequality (15) that

$$
\dot{g}_{\epsilon}(t) \leq \gamma(t) g_{\epsilon}(t)+\alpha(t) g_{\epsilon}^{p}(t-\tau)+\beta(t), \quad t \geq 0, \quad \epsilon>0 .
$$

Since $g(t)=\lim _{\epsilon \rightarrow 0} g_{\epsilon}(t)$ by (13), and $\lim _{\epsilon \rightarrow 0} \dot{g}_{\epsilon}(t)=\dot{g}(t)$, inequality (11a) follows from (16) by letting $\epsilon \rightarrow 0$. Lemma 2.1 is proved.

Consider the following delay differential equation

$$
\begin{aligned}
& \dot{h}(t)=\gamma(t) h(t)+\alpha(t) h^{p}(t-\tau)+\beta(t), \quad t \geq 0, \\
& h(t)=w(t), \quad-\tau \leq t \leq 0 .
\end{aligned}
$$

We have the following comparison lemma. 
Lemma 2.2. Let $g(t)$ solve inequality (7) and $h(t)$ solve Equation (17). Then

$$
g(t) \leq h(t), \quad \forall t \in[0, \tilde{T}),
$$

where $[-\tau, \tilde{T})$ is the maximal interval of the existence of $h(t)$.

Proof. Let $h_{n}(t)$ be the solution to the equation

$$
\begin{aligned}
& \dot{h}_{n}(t)=\gamma(t) h_{n}(t)+\alpha(t) h_{n}^{p}(t-\tau)+\beta(t)+\frac{1}{n}, \quad t \geq 0, \quad n=1,2, \ldots . . \\
& h_{n}(t)=w(t), \quad-\tau \leq t \leq 0 .
\end{aligned}
$$

Since $g(t)=w(t)=h_{n}(t), \forall t \in[-\tau, 0]$, it follows from (7a) and (18a) that

$$
\begin{aligned}
g^{\prime}(0) & \leq \gamma(0) g(0)+\alpha(0) g^{p}(-\tau)+\beta(0) \\
& =\gamma(0) h_{n}(0)+\alpha(0) h_{n}^{p}(-\tau)+\beta(0)=h_{n}^{\prime}(0)-\frac{1}{n}<h_{n}^{\prime}(0) .
\end{aligned}
$$

This and the equality $g(0)=h_{n}(0)$ imply the existence of $\delta>0$ such that $g(t)<h_{n}(t), \forall t \in(0, \delta)$. Let $T>0$ be the largest value such that

$$
g(t)<h_{n}(t), \quad \forall t \in(0, T) .
$$

We claim that $T=T_{n}$ where $\left[-\tau, T_{n}\right)$ is the maximal interval of the existence of $h_{n}(t)$.

To prove this claim, assume the contrary. Then $T<T_{n}$ and $h_{n}(T)=g(T)$, by the continuity of $g(t)$ and $h_{n}(t)$ and the definition of $T$. Since $g(t)<h_{n}(t), \forall t \in(0, T)$, and $g(T)=h_{n}(T)$, one concludes that

$$
\dot{g}(T) \geq \dot{h}_{n}(T) .
$$

On the other hand, from (7a), (18a), the equality $g(T)=h_{n}(T)$, and the inequality $g(T-\tau) \leq h(T-\tau)$, we have

$$
\begin{aligned}
\dot{g}(T) & \leq \gamma(T) g(T)+\alpha(T) g^{p}(T-\tau)+\beta(T) \\
& =\gamma(T) h_{n}(T)+\alpha(T) h_{n}^{p}(T-\tau)+\beta(T)=\dot{h}_{n}(T)-\frac{1}{n}<\dot{h}(T) .
\end{aligned}
$$

This contradicts to inequality (19) and implies that $T=T_{n}$, i.e.,

$$
g(t)<h_{n}(t), \quad \forall t \in\left(0, T_{n}\right) .
$$

Since $h(t)=\lim _{n \rightarrow \infty} h_{n}(t)$ and $\lim _{n \rightarrow \infty} T_{n}=\tilde{T}$, one gets $g(t) \leq h(t), \forall t \in[0, \tilde{T})$. Lemma 2.2 is proved.

Lemma 2.2 says that for any solution $g(t) \geq 0$ to inequality (7) one has $0 \leq g(t) \leq h(t)$. Thus, the global existence and boundedness of $h(t)$ imply the global existence and boundedness of $g(t)$. Therefore, to study the global existence and boundedness of $g(t)$, we will study the global existence and boundedness of the solution $h(t)$ to Equation (17).

Let us consider Equation (17). Since the functions $h(t), \alpha(t)$, and $f(t)$ are nonnegative on $\mathbb{R}_{+}$, it follows from (17a) that

$$
\dot{h}(t) \geq \gamma(t) h(t), \quad t \geq 0 .
$$

This inequality is equivalent to

$$
\frac{d}{d t}\left[h(t) e^{-\int_{0}^{t} \gamma(\xi) d \xi}\right] \geq 0, \quad t \geq 0
$$

Integrate this inequality from $t-\tau$ to $t$ to get

$$
h(t) e^{-\int_{0}^{t} \gamma(\xi) d \xi} \geq h(t-\tau) e^{-\int_{0}^{t-\tau} \gamma(\xi) d \xi}, \quad t \geq \tau .
$$

Thus,

$$
h(t) \sigma(t) \geq h(t-\tau), \quad \sigma(t):=e^{-\int_{t-\tau}^{t} \gamma(\xi) d \xi}, \quad t \geq \tau .
$$

This and inequality (17a) imply

$$
\dot{h}(t) \leq \gamma(t) h(t)+\alpha(t) \sigma^{p}(t) h^{p}(t)+\beta(t), \quad t \geq \tau .
$$

It follows from Equation (17a) that

$$
\frac{d}{d t}[h(t) \nu(t)]=\nu(t) \alpha(t) h^{p}(t-\tau)+\nu(t) \beta(t), \quad t \geq 0, \quad \nu(t):=e^{-\int_{0}^{t} \gamma(\xi) d \xi} .
$$


Integrate this equation from 0 to $\tau$ to get

$$
h(\tau) \nu(\tau)-h(0) \nu(0)=\int_{0}^{\tau}\left[\alpha(\xi) \nu(\xi) h^{p}(\xi-\tau)+\beta(\xi) \nu(\xi)\right] d \xi .
$$

This and the relation $\nu(0)=1$ imply

$$
h(\tau)=\frac{h(0)}{\nu(\tau)}+\frac{\int_{0}^{\tau}\left[\alpha(\xi) \nu(\xi)\|v(\xi-\tau)\|^{p}+\beta(\xi) \nu(\xi)\right] d \xi}{\nu(\tau)} .
$$

From (21) and (22) one concludes that the solution $h(t)$ to Equation (17) satisfies the following inequality

$$
\begin{aligned}
& \dot{h}(t) \leq \gamma(t) h(t)+\alpha(t) \sigma^{p}(t) h^{p}(t)+\beta(t), \quad t \geq \tau, \quad h(t) \geq 0, \\
& h(\tau)=h_{\tau}
\end{aligned}
$$

where

$$
h_{\tau}:=\frac{\|v(0)\|}{\nu(\tau)}+\frac{\int_{0}^{\tau}\left[\alpha(\xi) \nu(\xi)\|v(\xi-\tau)\|^{p}+\beta(\xi) \nu(\xi)\right] d \xi}{\nu(\tau)} .
$$

By our assumptions (3) and (4) it follows that $\alpha(t)$ and $\beta(t)$ are positive. Also $\nu(t)>0$. Therefore $h_{\tau}>0$. The function $\sigma(t)$, defined in (20), is also positive.

The following lemma gives a sufficient condition for the solution to Equation (17) to exist globally.

Lemma 2.3. Let $h(t)$ be a solution to Equation (17). Assume that

$$
\begin{gathered}
\frac{1}{\left[h_{\tau} \nu(\tau)+\omega\right]^{p-1}}>(p-1) \int_{\tau}^{\infty} \frac{\alpha(\xi) \sigma^{p}(\xi)}{\nu^{p-1}(\xi)} d \xi, \quad \nu(t):=e^{-\int_{0}^{t} \gamma(\xi) d \xi}, \\
\frac{\beta(t) \nu^{p}(t)}{\alpha(t) \sigma^{p}(t)} \leq \omega^{p}, \quad t \geq \tau, \quad \omega=\text { const }>0,
\end{gathered}
$$

where $h_{\tau}$ is defined in (24). Then $h(t)$ exists globally and satisfies the estimate

$$
h(t) \leq \frac{\left(\frac{1}{\left[h_{\tau} \nu(\tau)+\omega\right]^{1-p}-(p-1) \int_{\tau}^{t} \frac{\alpha(\xi) \sigma^{p}(\xi)}{\nu^{-1}(\xi)} d \xi}\right)^{\frac{1}{p-1}}-\omega}{\nu(t)}, \quad t \geq \tau .
$$

Remark 2.1. It follows from inequality (25) that the right-hand side of (27) is well-defined for all $t \geq \tau$. Inequality (25) is equivalent to

$$
\omega<\frac{1}{\left[(p-1) \int_{\tau}^{\infty} \frac{\alpha(\xi) \sigma^{p}(\xi)}{\nu^{-1}(\xi)} d \xi\right]^{\frac{1}{p-1}}}-h_{\tau} \nu(\tau) .
$$

Moreover, inequality (26) is equivalent to

$$
\left(\frac{\beta(t)}{\alpha(t)}\right)^{\frac{1}{p}} e^{-\int_{0}^{t-\tau} \gamma(\xi) d \xi} \leq \omega, \quad t \geq \tau .
$$

Thus, for the existence of $\omega$ satisfying both (25) and (26), it suffices to assume that

$$
\sup _{t \geq \tau}\left(\frac{\beta(t)}{\alpha(t)}\right)^{\frac{1}{p}} e^{-\int_{0}^{t-\tau} \gamma(\xi) d \xi}<\frac{1}{\left[(p-1) \int_{\tau}^{\infty} \frac{\alpha(\xi) \sigma^{p}(\xi)}{\nu^{p-1}(\xi)} d \xi\right]^{\frac{1}{p-1}}}-h_{\tau} \nu(\tau) .
$$

Given the function $\gamma(t)$ and the numbers $p, \tau, h_{\tau}$ and $\nu(\tau)$, inequality (28) holds true if $\alpha(t)$ and $\beta(t) / \alpha(t)$ are sufficiently small. In this case, one can choose

$$
\omega:=\sup _{t \geq \tau}\left(\frac{\beta(t)}{\alpha(t)}\right)^{\frac{1}{p}} e^{-\int_{0}^{t-\tau} \gamma(\xi) d \xi} .
$$

In the case when $f(t)$ is absent from Equation (1), i.e., $\beta(t)=0$, then inequality (28) becomes

$$
h_{\tau} \nu(\tau)<\frac{1}{\left[(p-1) \int_{\tau}^{\infty} \frac{\alpha(\xi) \sigma^{p}(\xi)}{\nu^{p-1}(\xi)} d \xi\right]^{\frac{1}{p-1}}}
$$

and one can choose $\omega=0$. 
Proof of Lemma 2.3. Since $h(t)$ is the solution to (17), it satisfies inequality (23a). From (23a) and (26), one gets

$$
\begin{aligned}
\dot{h} & \leq \gamma(t) h(t)+\frac{\alpha(t) \sigma^{p}(t)}{\nu^{p}(t)}\left[\nu^{p}(t) h^{p}(t)+\frac{\beta(t) \nu^{p}(t)}{\alpha(t) \sigma^{p}(t)}\right] \\
& \leq \gamma(t) h(t)+\frac{\alpha(t) \sigma^{p}(t)}{\nu^{p}(t)}\left[\nu^{p}(t) h^{p}(t)+\omega^{p}\right] \\
& \leq \gamma(t) h(t)+\frac{\alpha(t) \sigma^{p}(t)}{\nu^{p}(t)}[\nu(t) h(t)+\omega]^{p}, \quad t \geq \tau, \quad p>1 .
\end{aligned}
$$

Here, the inequality $a^{p}+b^{p} \leq(a+b)^{p}, a \geq 0, b \geq 0, p>1$, was used. Inequality (29) can be written as

$$
\frac{d}{d t}(h(t) \nu(t)+\omega) \leq \frac{\alpha(t) \sigma^{p}(t)}{\nu^{p-1}(t)}[\nu(t) h(t)+\omega]^{p}, \quad t \geq \tau .
$$

Thus,

$$
\frac{1}{1-p} \frac{d}{d t}[h(t) \nu(t)+\omega]^{1-p} \leq \frac{\alpha(t) \sigma^{p}(t)}{\nu^{p-1}(t)}, \quad t \geq \tau
$$

Integrate inequality (30) from $\tau$ to $t$ to get

$$
\frac{[h(t) \nu(t)+\omega]^{1-p}-[h(\tau) \nu(\tau)+\omega]^{1-p}}{1-p} \leq \int_{\tau}^{t} \frac{\alpha(\xi) \sigma^{p}(\xi)}{\nu^{p-1}(\xi)} d \xi, \quad t \geq \tau
$$

This implies

$$
[h(t) \nu(t)+\omega]^{1-p} \geq[h(\tau) \nu(\tau)+\omega]^{1-p}-(p-1) \int_{\tau}^{t} \frac{\alpha(\xi) \sigma^{p}(\xi)}{\nu^{p-1}(\xi)} d \xi, \quad t \geq \tau .
$$

It follows from (25) that the right-hand side of inequality (31) is positive. Thus, from (31) one gets

$$
[h(t) \nu(t)+\omega]^{p-1} \leq \frac{1}{\left[h_{\tau} \nu(\tau)+\omega\right]^{1-p}-(p-1) \int_{\tau}^{t} \frac{\alpha(\xi) \sigma^{p}(\xi)}{\nu^{p-1}(\xi)} d \xi}, \quad t \geq \tau .
$$

Inequality (27) follows from (32). Lemma 2.3 is proved.

Theorem 2.1. Let Assumption A hold. Assume that

$$
\omega:=\sup _{t \geq \tau}\left(\frac{\beta(t)}{\alpha(t)}\right)^{\frac{1}{p}} e^{-\int_{0}^{t-\tau} \gamma(\xi) d \xi}<\frac{1}{\left[(p-1) \int_{\tau}^{\infty} \frac{\alpha(\xi) \sigma^{p}(\xi)}{\nu^{p-1}(\xi)} d \xi\right]^{\frac{1}{p-1}}}-h_{\tau} \nu(\tau)
$$

where

$$
h_{\tau}:=\frac{\|v(0)\|}{\nu(\tau)}+\frac{\int_{0}^{\tau}\left[\alpha(\xi) \nu(\xi)\|v(\xi-\tau)\|^{p}+\beta(\xi) \nu(\xi)\right] d \xi}{\nu(\tau)},
$$

$\sigma(t):=e^{-\int_{\tau}^{t} \gamma(\xi) d \xi}$ and $\nu(t):=e^{-\int_{0}^{t} \gamma(\xi) d \xi}$. Then the solution to problem (1) exists globally and

$$
\|u(t)\| \leq \frac{1}{\nu(t)}\left[\left(\frac{1}{\left[h_{\tau} \nu(\tau)+\omega\right]^{1-p}-(p-1) \int_{\tau}^{t} \frac{\alpha(\xi) \sigma^{p}(\xi)}{\nu^{p-1}(\xi)} d \xi}\right)^{\frac{1}{p-1}}-\omega\right], \quad t \geq \tau .
$$

In addition, if

$$
M:=\sup _{t \geq 0} \int_{0}^{t} \gamma(\xi) d \xi<\infty,
$$

then the solution $u(t)$ is bounded.

If

$$
\lim _{t \rightarrow \infty} \int_{0}^{t} \gamma(\xi) d \xi=-\infty
$$

then

$$
\lim _{t \rightarrow \infty} u(t)=0 .
$$

Proof. Let $u(t)$ be the solution to (1) and $g(t):=\|u(t)\|$. Then from Lemma 2.2 one has

$$
g(t) \leq h(t), \quad t \geq 0 .
$$


It follows from (33) and Remark 2.1 that inequalities (25) and (26) hold. Using Lemma 2.3 one gets

$$
h(t) \leq \frac{1}{\nu(t)}\left[\left(\frac{1}{\left[h_{\tau} \nu(\tau)+\omega\right]^{1-p}-(p-1) \int_{\tau}^{t} \frac{\alpha(\xi) \sigma^{p}(\xi)}{\nu^{p-1}(\xi)} d \xi}\right)^{\frac{1}{p-1}}-\omega\right], \quad t \geq \tau .
$$

Therefore,

$$
\|u(t)\|=g(t) \leq h(t) \leq \frac{\left(\frac{1}{\left[h_{\tau} \nu(\tau)+\omega\right]^{1-p}-(p-1) \int_{\tau}^{t} \frac{\alpha(\xi) \sigma^{p}(\xi)}{\nu^{p-1}(\xi)} d \xi}\right)^{\frac{1}{p-1}}-\omega}{\nu(t)}, \quad t \geq \tau .
$$

Thus, inequality (34) holds.

Inequality (38) implies

$$
\|u(t)\| \leq \frac{C}{\nu(t)}=C e^{\int_{0}^{t} \gamma(\xi) d \xi}, \quad t \geq \tau,
$$

where

$$
C:=\left(\frac{1}{\left[h_{\tau} \nu(\tau)+\omega\right]^{1-p}-(p-1) \int_{\tau}^{\infty} \frac{\alpha(\xi) \sigma^{p}(\xi)}{\nu^{p-1}(\xi)} d \xi}\right)^{\frac{1}{p-1}}-\omega .
$$

Inequality (39) and Assumption A imply that the solution $u(t)$ exists globally.

If inequality (35) holds, then

$$
e^{\int_{0}^{t} \gamma(\xi) d \xi} \leq e^{M}, \quad \forall t \geq 0 .
$$

This and inequality (39) imply

$$
\|u(t)\| \leq C e^{M}, \quad t \geq \tau .
$$

Thus, the solution $u(t)$ is bounded on $\mathbb{R}_{+}$.

If relation (36) holds, then one gets

$$
\lim _{t \rightarrow \infty} e^{\int_{0}^{t} \gamma(\xi) d \xi}=0 .
$$

Relation (37) follows from inequality (39) and formula (40). Theorem 2.1 is proved.

It follows from (33) in Theorem 2.1 that if the right-hand side of (33) is positive, then one can obtain the global existence and boundedness of $u(t)$ by choosing $f(t)$ so that $\beta(t):=\|f(t)\|$ is sufficiently small. This raises the question: Given $\beta(t):=\|f(t)\|$, is this possible to 'control' $\alpha(t)$ so that the global existence and boundedness of $u(t)$ are still guaranteed? We will address this question in the following results.

Lemma 2.4. Let $h(t)$ be the solution to Equation (17) and

$$
\zeta(t):=\frac{h(\tau) \nu(\tau)}{\nu(t)}+\frac{\int_{\tau}^{t} \beta(\xi) \nu(\xi) d \xi}{\nu(t)}, \quad t \geq \tau .
$$

Assume that

$$
\alpha(t) \sigma^{p}(t) \leq \frac{(q-1) \beta(t)}{[q \zeta(t)]^{p}}, \quad t \geq \tau, \quad q>1
$$

Then

$$
h(t) \leq q \zeta(t), \quad t \geq \tau .
$$

Proof. From Equation (41) with $t=\tau$ and the assumption $q>1$, one gets

$$
h(\tau)=\zeta(\tau)<q \zeta(\tau)
$$

It follows from the continuity of $\zeta(t)$ and $h(t)$ that there exists $\theta>0$ such that

$$
h(t) \leq q \zeta(t), \quad \tau \leq t \leq \tau+\theta .
$$

Let $T>0$ be the largest real value such that

$$
h(t) \leq q \zeta(t), \quad \tau \leq t \leq T .
$$

We claim that $T=\infty$. Assume the contrary. Then $T$ is finite and by the definition of $T$ we have

$$
h(T)=q \zeta(T) .
$$


Since $h(t)$ is the solution to (17), it satisfies inequality (23a). It follows from inequalities (23a), (44), and (42) that

$$
\begin{aligned}
\dot{h}(t) & \leq \gamma(t) h(t)+\alpha(t) \sigma^{p}(t)[q \zeta(t)]^{p}+\beta(t) \\
& \leq \gamma(t) h(t)+(q-1) \beta(t)+\beta(t)=\gamma(t) h(t)+q \beta(t), \quad \tau \leq t \leq T .
\end{aligned}
$$

From (46) one gets

$$
\frac{d}{d t}[\nu(t) h(t)] \leq q \nu(t) \beta(t), \quad \tau \leq t \leq T .
$$

Integrate this inequality from $\tau$ to $t$ to get

$$
\nu(t) h(t)-\nu(\tau) h(\tau) \leq q \int_{\tau}^{t} \beta(\xi) \nu(\xi) d \xi, \quad \tau \leq t \leq T .
$$

This and (41) imply

$$
\begin{aligned}
h(t) & \leq \frac{\nu(\tau) h(\tau)}{\nu(t)}+\frac{q \int_{\tau}^{t} \beta(\xi) \nu(\xi) d \xi}{\nu(t)} \\
& <q\left(\frac{\nu(\tau) h(\tau)}{\nu(t)}+\frac{\int_{\tau}^{t} \beta(\xi) \nu(\xi) d \xi}{\nu(t)}\right)=q \zeta(t), \quad \tau \leq t \leq T .
\end{aligned}
$$

Therefore,

$$
h(T)<q \zeta(T) .
$$

This contradicts to Equation (45). The contradiction implies that $T=\infty$, i.e., inequality (43) holds. Lemma 2.4 is proved.

Theorem 2.2. Let Assumption A hold. Assume that

$$
h(\tau)>0, \quad \alpha(t) \sigma^{p}(t) \leq \frac{(q-1) \beta(t)}{[q \zeta(t)]^{p}}, \quad t \geq \tau, \quad q>1,
$$

where

$$
\sigma(t)=e^{-\int_{t-\tau}^{t} \gamma(\xi) d \xi}, \quad \zeta(t)=\frac{h(\tau) \nu(\tau)}{\nu(t)}+\frac{\int_{\tau}^{t} \beta(\xi) \nu(\xi) d \xi}{\nu(t)}, \quad t \geq \tau, \quad \nu(t)=e^{-\int_{0}^{t} \gamma(\xi) d \xi} .
$$

Then the solution u(t) to Equation (1) exists globally.

If

$$
M=\sup _{t \geq \tau} \int_{\tau}^{t} \gamma(\xi) d \xi<\infty, \quad \int_{\tau}^{\infty} \beta(\xi) \nu(\xi) d \xi<\infty,
$$

then the solution $u(t)$ to problem (1) is bounded.

If

$$
\lim _{t \rightarrow \infty} \int_{\tau}^{t} \gamma(\xi) d \xi=-\infty
$$

and either

$$
\int_{\tau}^{\infty} \beta(\xi) \nu(\xi) d \xi<\infty \quad \text { or } \quad \lim _{t \rightarrow \infty} \frac{\beta(t)}{\gamma(t)}=0,
$$

then

$$
\lim _{t \rightarrow \infty} u(t)=0 .
$$

Proof. Let $g(t)=\|u(t)\|$. Then from Lemma 2.2 we have

$$
\|u(t)\|=g(t) \leq h(t), \quad t \geq \tau .
$$

This and Lemma 2.4 imply

$$
\|u(t)\| \leq h(t) \leq q \zeta(t)=\frac{h(\tau) \nu(\tau)}{\nu(t)}+\frac{\int_{\tau}^{t} \beta(\xi) \nu(\xi) d \xi}{\nu(t)}, \quad t \geq \tau .
$$

It follows from (52) and Assumption A that the solution $u(t)$ to Equation (1) exists globally.

If relation (48) hold, then

$$
\nu(t)=e^{-\int_{0}^{t} \gamma(\xi) d \xi} \geq e^{-M}, \quad \forall t \geq \tau .
$$

This, inequality (52), and the second inequality in (48) imply

$$
\|u(t)\| \leq e^{M}\left(h(\tau) \nu(\tau)+\int_{0}^{\infty} \beta(\xi) \nu(\xi) d \xi\right)<\infty .
$$


This means the solution $u(t)$ is bounded on $\mathbb{R}_{+}$.

If relation (49) holds, then one gets

$$
\lim _{t \rightarrow \infty} \nu(t)=\lim _{t \rightarrow \infty} e^{-\int_{\tau}^{t} \gamma(\xi) d \xi}=\infty
$$

We claim that

$$
\lim _{t \rightarrow \infty} \frac{\int_{\tau}^{t} \beta(\xi) \nu(\xi) d \xi}{\nu(t)}=0 .
$$

Indeed, if $\int_{\tau}^{\infty} \beta(\xi) \nu(\xi) d \xi<\infty$, then relation (54) follows from (53). If $\int_{\tau}^{\infty} \beta(\xi) \nu(\xi) d \xi=\infty$, then relation (54) follows from the second relation in (50) and L'Hospital's rule.

From inequality (52) and equalities (53) and (54) one obtains

$$
\lim _{t \rightarrow \infty}\|u(t)\| \leq \lim _{t \rightarrow \infty} \frac{h(\tau) \nu(\tau)}{\nu(t)}+\lim _{t \rightarrow \infty} \frac{\int_{\tau}^{t} \beta(\xi) \nu(\xi) d \xi}{\nu(t)}=0 .
$$

Thus, equality (51) holds. Theorem 2.2 is proved.

If $f \equiv 0$, or equivalently $\beta(t) \equiv 0$, then the second inequality in (47) implies that $\alpha(t) \equiv 0$. Thus, the result in Theorem 2.2 is not very interesting if $f \equiv 0$. However, if the functions $\gamma(t)$ and $\beta(t)$ satisfy either inequalities (48) or inequalities (49) and (50), then the global existence and boundedness of the solution $u(t)$ can be obtained if $\alpha(t)$ is sufficiently small so that the second inequality in (47) holds.

Let us consider Equation (1) with $f=0$ :

$$
\begin{array}{rlrl}
\dot{u} & =A(t) u+G(t, u(t-\tau)), \quad t \geq 0, \quad \dot{u}:=\frac{d u}{d t}, & \\
u(t) & =v(t), \quad-\tau \leq t \leq 0, \quad v(t) \in C([-\tau, 0] ; \mathcal{H}), \quad \tau=\text { const }>0 .
\end{array}
$$

For Equation (55) we use the following assumption:

Assumption B: The equation

$$
\begin{aligned}
\dot{u} & =A(t) u+G(t, u(t-\tau)), \quad t \geq a, \quad \dot{u}:=\frac{d u}{d t}, \\
u(t) & =v_{a}(t), \quad a-\tau \leq t \leq a, \quad v_{a} \in C([a-\tau, a] ; \mathcal{H}), \quad \tau=\text { const }>0,
\end{aligned}
$$

has a unique local solution for all $a \geq 0$ and $v_{a} \in C([a-\tau, a] ; \mathcal{H})$.

Using Theorem 2.1 with $\omega=0$ for Equation (55) we have the following corollary:

Corollary 2.1. Let Assumption $\mathbf{B}$ hold and $u(t)$ be the solution to (55). Assume that

$$
\begin{array}{ll}
\frac{1}{\left[\tilde{h}_{\tau} \nu(\tau)\right]^{p-1}}>(p-1) \int_{\tau}^{\infty} \frac{\alpha(\xi) \sigma^{p}(\xi)}{\nu^{p-1}(\xi)} d \xi, & \sigma(t):=e^{-\int_{t-\tau}^{t} \gamma(\xi) d \xi}, \\
\tilde{h}_{\tau}:=\frac{\|v(0)\|}{\nu(\tau)}+\frac{\int_{0}^{\tau} \alpha(\xi) \nu(\xi)\|v(\xi-\tau)\|^{p} d \xi}{\nu(\tau)}, & \nu(t)=e^{-\int_{0}^{t} \gamma(\xi) d \xi} .
\end{array}
$$

Then the solution to problem (55) exists globally and

$$
\|u(t)\| \leq \frac{1}{\nu(t)}\left(\frac{1}{\left[\tilde{h}_{\tau} \nu(\tau)\right]^{1-p}-(p-1) \int_{\tau}^{t} \frac{\alpha(\xi) \sigma^{p}(\xi)}{\nu^{p-1}(\xi)} d \xi}\right)^{\frac{1}{p-1}}, \quad t \geq \tau .
$$

In addition, if

$$
\sup _{t \geq 0} \int_{0}^{t} \gamma(\xi) d \xi<\infty
$$

then the solution $u(t)$ is bounded.

If

$$
\lim _{t \rightarrow \infty} \int_{0}^{t} \gamma(\xi) d \xi=-\infty
$$

then

$$
\lim _{t \rightarrow \infty} u(t)=0 .
$$


If $\|v(t)\|$ is sufficiently small on $[-\tau, 0]$, then number

$$
\tilde{h}_{\tau}:=\frac{\|v(0)\|}{\nu(\tau)}+\frac{\int_{0}^{\tau} \alpha(\xi) \nu(\xi)\|v(\xi-\tau)\|^{p} d \xi}{\nu(\tau)}
$$

is sufficiently small and, therefore, the quotient $1 /\left[\tilde{h}_{\tau} \nu(\tau)\right]^{p-1}$ is sufficiently large. Thus, inequality (57) holds if $\|v(t)\|$ is sufficiently small on $[-\tau, 0]$. If relation (60) holds, then the function $\frac{1}{\nu(t)}=e^{\int_{0}^{t} \gamma(\xi) d \xi}$ is bounded. Therefore, the right-hand side of (59) can be made arbitrarily small by making $\tilde{h}_{\tau}$ sufficiently small. Hence, it follows from (59) that $\sup _{t \geq 0}\|u(t)\|$ can be made arbitrarily small by making $\|v(t)\|$ sufficiently small on $[-\tau, 0]$. If (57) and (61) hold, then relation (62) holds. These arguments and Corollary 2.1 yield the following result:

Corollary 2.2. Let Assumption B hold. Assume that

If

$$
\int_{\tau}^{\infty} \frac{\alpha(\xi) \sigma^{p}(\xi)}{\nu^{p-1}(\xi)} d \xi<\infty, \quad \nu(t):=e^{-\int_{0}^{t} \gamma(\xi) d \xi}, \quad \sigma(t):=e^{-\int_{t-\tau}^{t} \gamma(\xi) d \xi}
$$

$$
\sup _{t \geq 0} \int_{0}^{t} \gamma(\xi) d \xi<\infty
$$

then the equilibrium solution $u=0$ to Equation (55) is Lyapunov stable. If

$$
\lim _{t \rightarrow \infty} \int_{0}^{t} \gamma(\xi) d \xi=-\infty
$$

then the equilibrium solution $u=0$ to problem (55) is asymptotically stable, i.e., $\lim _{t \rightarrow \infty} u(t)=0$ if $\|u(t)\|$ is sufficiently small on $[-\tau, 0]$.

Using arguments similar to the ones in [9], one obtains the following result:

Lemma 2.5. If there exists a function $\mu(t)>0$, defined for all $t \geq-\tau$, such that

$$
\begin{aligned}
\alpha(t) \frac{\mu(t)}{\mu^{p}(t-\tau)}+\beta(t) \mu(t) & \leq-\gamma(t)-\frac{\dot{\mu}(t)}{\mu(t)}, \quad t \geq 0, \\
\|u(t)\| & \leq \frac{1}{\mu(t)}, \quad t \in[-\tau, 0],
\end{aligned}
$$

then the solution to Equation (1) exists for all $t \geq 0$ and

$$
\|u(t)\| \leq \frac{1}{\mu(t)}, \quad t \geq 0
$$

As we have mentioned earlier, although results similar to Lemma 2.5 are quite general, their applications rely on the existence of $\mu(t)>0$ satisfying inequality (63). Theoretically, Lemma 2.5 is applicable as long as the solution to Equation (17) exists globally. If this is the case, then one can take $\mu(t)=\frac{1}{h(t)}$ and this function satisfies (63). However, it is not known if there is an explicit formula for the solution $h(t)$ to Equation (17). Moreover, if $\alpha(t), \beta(t)$, and $\gamma(t)$ are some general functions, it is not easy to find $\mu(t)$ which solves inequality (63).

\section{Conclusion}

Several stability results of the solution to Equation (1) under non-classical assumptions on the operator $A(t)$ have been proposed and proved. In classical results it is often assumed that the function $\gamma(t)$ in (2) satisfies the inequality $\gamma(t) \leq \gamma_{0}<$ 0 . Our results allow the function $\gamma(t)$ take both positive and negative values on $[0, \infty)$. Stability of solutions to abstract evolution equations with delay was also studied in [9]. The advantage of our results compared to those in [9] is: one does not have to find a function $\mu(t)$ which solves inequalities in (9). Moreover, our results are applicable to more general equations than those in [9].

\section{References}

[1] O. Aribo, M. Hbid, E. Ait Dads (Eds.), Delay Differential Equations and Applications, NATO Science Series II: Mathematics, Physics and Chemistry (Vol. 206), Springer, Dordrecht, 2006.

[2] T. Faria, Global attractivity in scalar delayed differential equations with applications to population models, J. Math. Anal. Appl. 289 (2004) 35-54.

[3] J. Hale, S. Verduyn Lunel, Introduction to Functional Differential Equations, Springer, Berlin, 1993.

[4] N. S. Hoang, Stability results of some abstract evolution equations, Differ. Equ. Appl. 6 (2014) 417-428.

[5] V. Kolmanovskii, A. D. Myshkis, Introduction to the Theory and Applications of Functional-Differential Equations, Kluwer, Dordrecht, 1999.

[6] A. G. Ramm, Asymptotic stability of solutions to abstract differential equations, J. Abstr. Differ. Equ. Appl. 1 (2010) 27-34.

[7] A. G. Ramm, Stability of solutions to some evolution problems, Chaotic Model. Simul. 1 (2011) 17-27.

[8] A. G. Ramm, A stability result for abstract evolution problems, Math. Methods Appl. Sci., 36 (2012) 422-426.

[9] A. G. Ramm, Stability of solutions to abstract evolution equations with delay, J. Math. Anal. Appl. 396 (2012) 523-527.

[10] A. G. Ramm, N. S. Hoang, Dynamical Systems Method and Applications: Theoretical Developments and Numerical Examples, Wiley, Hoboken, 2012. 